\title{
Uso del estiércol porcino sólido como abono orgánico en el cultivo del maíz chala
}

\author{
Use of pig solid manure as organic fertilizer in corn forage production
}

\author{
Luis Moreno Ayala ${ }^{1} \&$ José Cadillo Castro ${ }^{2 *}$ \\ * Autor de correspondencia
}

\section{Resumen}

La investigación tuvo como objetivo evaluar las bondades del estiércol porcino sólido como abono orgánico, medido a través del rendimiento forrajero, valor nutricional de la planta, propiedades fisicoquímicas del suelo post cosecha, costo de producción y beneficio neto del maíz chala. Para lo cual se planteó tres tratamientos: fertilizante químico (Control, T1), estiércol sólido (T2) y fertilizante químico + estiércol sólido (T3). El análisis de varianza para el rendimiento forrajero se realizó a través de un Diseño de Bloques Completamente al Azar (DBCA) y la prueba de Duncan ( $<<0,05)$; el valor nutricional de la planta y las propiedades fisicoquímicas del suelo se obtuvo a través de análisis de laboratorio; el costo de producción y el beneficio neto a través de un análisis económico. El rendimiento forrajero resulto estadísticamente no significativo ( $>0,05)$, entre los tres tratamiento; sin embargo se tuvo mayores valores con el tratamiento $\mathrm{T} 2,1,08 \mathrm{~kg}$ de peso fresco por planta y 73,8 t/ha, $5 \mathrm{t}$ más que el tratamiento $\mathrm{T} 1$. El mayor valor nutricional de la planta, se obtuvo con el tratamiento T3, 10,5\% proteína cruda, 1,6\% grasa, 25,4\% fibra cruda y 54,8 \% fibra detergente neutra. Mejores propiedades fisicoquímicas del suelo post cosecha se obtuvo con el tratamiento T2, 2,56 \% materia orgánica, 59,4 ppm fosforo y 230 ppm potasio. La mayor rentabilidad de la producción de forraje se obtuvo con el tratamiento T2, S/ 3,858.60 de costo de producción y S/ 4,270.40 de beneficio neto por hectárea.

Palabras clave: Estiércol sólido; abono orgánico; valor nutritivo; suelo; rentabilidad.

\begin{abstract}
The objective of the research was to evaluate the benefits of pig solid manure as an organic fertilizer, measured through forage yield, plant nutritional value, physicochemical properties of post-harvest soil, cost of production and net benefit of forage maize. For which three treatments were proposed: chemical fertilizer (Control, T1), solid manure (T2) and chemical fertilizer + solid manure (T3). Analysis of variance for forage yield was performed through a completely randomized block design (DBCA) and Duncan's test $(\mathrm{p}<0,05)$; the nutritional value of the plant and the physicochemical properties of the soil were obtained through laboratory analysis; the cost of production and the net benefit through an economic analysis. The forage yield was statistically non-significant ( $p>0,05)$, among the three treatments; however, higher values were obtained with the T2 treatment, 1, $08 \mathrm{~kg}$ fresh weight per plant and 73, $8 \mathrm{t}$ per hectare, with a difference of five $\mathrm{t}$ with reference to the lowest value of the T1 treatment. The highest nutritional value of the plant was obtained with T3 treatment, 10,5\% crude protein, 1,6\% fat, 25,4 \% crude fiber and 54,8 \% neutral detergent fiber. Better physicochemical properties of post-harvest soil were obtained with T2 treatment, 2, 56 \% organic matter, 59,4 ppm phosphorus and $230 \mathrm{ppm}$ potassium. The highest yield of forage production was obtained with the T2 treatment, S/ 3,858.60 cost of production and S/ 4,270. 40 net benefit per hectare.
\end{abstract}

Keywords: Solid manure; Organic fertilizer; Nutritive value; Soil; Profitability.

\section{Introducción}

Según FAO (2 016) la carne roja de mayor consumo en el mundo es la carne de cerdo, cuya demanda en las últimas décadas ha tenido un fuerte incremento. Asimismo, indica que la producción porcina está distribuida prácticamente en todo el mundo, salvo en algunas regiones que por motivos religiosos y culturales no se consume carne de cerdo; y se caracteriza por la creciente dicotomía de los sistemas de producción; hay todavía una fuerte presencia de los sistemas tradicionales de subsistencia a pequeña escala, que convive con los sistemas industriales especializados.
Estos últimos se caracterizan por ser intensivos, a mediana y gran escala, y se concentran mayormente cerca a núcleos urbanos y a fuentes de insumos.

Según la Asociación Peruana de Porcicultores (2018) la producción de carne de cerdo el año 2017 fue de 208 mil toneladas, 4 \% más que el año 2 016; asimismo manifiesta que el consumo per cápita fue de $6,6 \mathrm{~kg}$. Estas cifras evidencian el incremento de la población y producción de carne de cerdo. En cuanto a la población de cerdos, según el INEI (2013) esta es de 2224300 cabezas, de los cuales el $67,2 \%$ son cerdos criollos y el $32,8 \%$ cerdos de razas 
mejoradas. Estas últimas se encuentran mayormente en la costa $(62,2 \%)$, cerca de zonas altamente pobladas, por ejemplo Lima Metropolitana (44\%), criados en sistemas intensivos.

Los sistemas intensivos de crianza se caracterizan porque los cerdos son criados en confinamiento, en corrales con piso de cemento (cuya limpieza demanda de abundante agua), se alimentan con alimentos balanceados y los cerdos son de alta genética; además en estos sistemas hay una gran concentración de animales en espacios reducidos y su nivel tecnológico de crianza va de medio a alto. Si bien estos sistemas permiten una mayor producción y mejoras sustanciales en la productividad, también traen consigo un aumento en el volumen del estiércol producido, principal desecho de la crianza intensiva del ganado, que si no se le maneja adecuadamente se puede convertir en una fuente de contaminación del suelo, agua y aire.

El estiércol porcino, de consistencia liquida, es la mezcla de heces, orina, agua de la limpieza de los corrales, más el alimento y agua que se desperdicia; tiene valor agronómico, ya que se puede usar como abono orgánico, para la producción de cultivos sin impactos ambientales significativos (Eghball et al., 2004). Si no es manejado adecuadamente, puede impactar negativamente al medio ambiente, perjudicando, el medio hídrico, atmosférico y suelo (Pinos et al., 2012).

Los abonos orgánicos se han utilizado desde tiempos remotos y su influencia sobre la fertilidad de los suelos ha sido demostrada ampliamente (Piccinini y Bortone, 1991). Las excretas animales son benéficas para los suelos debido a que los organismos del suelo descomponen la materia orgánica, lo que puede luego aumentar la capa arable, la aireación y la fertilidad, incrementar la capacidad de retención de agua y potencialmente reducir la erosión por viento y agua (Geohring \& Van Es, 1994).

El estiércol porcino, como tal, puede ser usado como abono orgánico; aunque también hay la posibilidad de separarlo en su fracción líquida y sólida. La primera puede ser usar para el riego y la segunda como "guano" para fertilizar los campos de cultivo. Esta opción es más amigable con el medio ambiente, da valor agregado a un residuo muchas veces no aprovechado, es más económico que los fertilizantes sintéticos y posibilita desarrollar actividades productivas sustentables. Bajo este concepto, se planteó el presente trabajo de investigación con el objetivo de evaluar el uso del estiércol sólido porcino como abono orgánico del maíz chala, medido a través del rendimiento forrajero (peso fresco de la planta, peso fresco por ha), valor nutricional de la planta, propiedades fisicoquímicas del suelo y un análisis económico.

\section{Materiales y métodos}

\section{Lugar de estudio y duración}

El estudio se realizó en el área agrícola de la empresa porcina Inversiones Analau S.A.C. ubicada en el km 32 de la panamericana sur, distrito de Pachacámac, provincia de Lima, a una altitud de $73 \mathrm{msnm}$, latitud sur $12^{\circ} 13^{\prime} 43^{\prime \prime}$ y longitud oeste $76^{\circ} 51^{\prime} 35^{\prime \prime}$. La duración fue de 6 meses, febrero a julio del 2016.

\section{Análisis de laboratorio}

\section{Composición química del estiércol sólido porcino.}

Se recolectó muestras del estercolero, lugar donde se deposita el estiércol de todas las áreas de la granja. Para obtener el estiércol sólido se utilizó una prensa, que separa los sólidos del líquido. Se hizo el análisis químico de la parte sólida del estiércol (Tabla 1) en el laboratorio de análisis de suelos de la Universidad Nacional Agraria La Molina (UNALM).

Tabla 1. Composición química del estiércol sólido porcino

\begin{tabular}{cccccccc}
\hline $\begin{array}{c}\mathrm{MO} \\
\%\end{array}$ & $\begin{array}{c}\text { Humedad } \\
\%\end{array}$ & $\mathrm{pH}$ & $\begin{array}{c}\mathrm{CE} \\
\mathrm{dS} / \mathrm{m}\end{array}$ & $\begin{array}{c}\mathrm{N} \\
\%\end{array}$ & $\begin{array}{c}\mathrm{P}_{2} \mathrm{O}_{5} \\
\mathrm{~K}_{2} \mathrm{O}\end{array}$ & $\begin{array}{c}\mathrm{CaO} \\
\%\end{array}$ \\
\hline 80.86 & 26.23 & 6.36 & 6.27 & 2.04 & 6.1 & 1.63 & 3.98 \\
$\mathrm{MgO}$ & $\mathrm{Na}$ & $\mathrm{Fe}$ & $\mathrm{Cu}$ & $\mathrm{Zn}$ & $\mathrm{Mn}$ & $\mathrm{B}$ & \\
$\%$ & $\%$ & $\mathrm{ppm}$ & $\mathrm{ppm}$ & $\mathrm{ppm}$ & $\mathrm{ppm}$ & $\mathrm{ppm}$ & \\
2.0 & 0.23 & 29 & 385 & 1580 & 488 & 1417 & \\
\hline
\end{tabular}

CE: Conductibilidad Eléctrica

MO: Materia Orgánica

\section{Propiedades fisicoquímicas del suelo}

Se tomó muestras de suelo antes de la siembra de todo el campo experimental para su respectivo análisis fisicoquímico (Tabla 2). Asimismo luego de la cosecha se tomaron muestras de suelo de cada uno de los tratamientos. Dichas muestras fueron analizadas en el laboratorio de análisis de suelos de la UNALM.

Tabla 2. Análisis fisicoquímico inicial del suelo

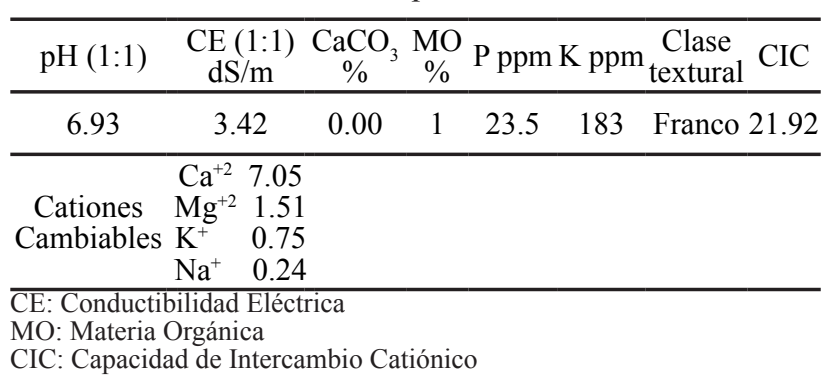

\section{Análisis nutricional de la planta}

Se utilizó el maíz PM-102, "Experimental 5". Para el análisis nutricional se tomó al azar 10 plantas por tratamiento, en el periodo vegetativo-grano pastoso, picadas y mezcladas homogéneamente. Se tomó $1 \mathrm{~kg}$ de muestra por tratamiento y fueron analizadas en el laboratorio de evaluación nutricional de alimentos de la UNALM.

\section{Tratamientos y distribución}

Se tuvo tres tratamientos: tratamiento control $\mathrm{T} 1$ (fertilizante químico), tratamiento T2 (estiércol sólido) y 
tratamiento T3 (fertilizante químico + estiércol sólido). Se utilizó 15 parcelas, cinco parcelas por tratamiento, distribuidos al azar. Cada parcela tuvo un área de $30 \mathrm{~m}^{2}$ $(6 \times 5 \mathrm{~m})$.

\section{Aplicación de los abonos}

Fertilizante químico.

Se aplicó la dosis 200-80-80 kg/ha de N, P, K respectivamente, en dos dosis. La primera se aplicó a los 10 días post siembra, dosis 100-80-80 kg/ha de N, P, K, y la segunda, a los 30 días post siembra, $100 \mathrm{~kg} / \mathrm{ha}$ de $\mathrm{N}$.

\section{Estiércol sólido}

Se aplicó el equivalente a 12 t/ha de estiércol sólido, por única vez, dos semanas antes de la siembra. La aplicación consistió en esparcir el abono en los surcos y mezclarlos con el suelo.

\section{Fertilizante químico más estiércol sólido}

Se aplicó el equivalente a 6 t/ha de estiércol sólido al suelo por única vez, dos semanas antes de la siembra y el fertilizante químico se aplicó en dos dosis, la primera a los 10 días post siembra, dosis de 50-40-40 kg/ha de N, P, K respectivamente y la segunda, a los 30 días post siembra, $50 \mathrm{~kg} / \mathrm{ha}$ de $\mathrm{N}$.

\section{Población y muestra}

Cada parcela tuvo aproximadamente 200 plantas, equivalente a 1000 plantas por tratamiento. Se tomaron 15 plantas de los surcos centrales de cada parcela, haciendo un total de 75 plantas seleccionadas aleatoriamente por tratamiento, con el propósito de medir el peso fresco de la planta.

\section{Diseño experimental}

Se utilizó el Diseño de Bloques Completamente al Azar (DBCA). El bloque se basó por el nivel de infiltración de agua. Las comparaciones de medias de los tratamientos se realizaron a través de la prueba de Duncan.

\section{Análisis económico}

Se evaluaron los costos de producción, ingresos netos e índice de rentabilidad.

\section{Resultados y discusión}

\section{$\underline{\text { Rendimiento forrajero }}$}

Peso Fresco de Planta. Los pesos obtenidos fueron: 1.01, 1.08 y $1.05 \mathrm{~kg}$ para los tratamientos T1, T2 y T3, respectivamente (Tabla 3); no existiendo diferencia estadística significativa $(\mathrm{p}>0.05)$ entre los tratamientos. Sin embargo se observa una tendencia, a mayor cantidad de estiércol, mayor peso por planta (70 g más en el tratamiento $\mathrm{T} 2$, con respecto al T1). En varias investigaciones (López et al., 2001; Álvarez, 2010; Sotomayor et al., 2017) se ha demostrado que los abonos orgánicos pueden reemplazar en su totalidad a los fertilizantes químicos, manteniendo e inclusive superándolos en la producción de maíz; porque entre otras cosas, mejoran las propiedades del suelo y aportan buena cantidad de materia orgánica.

Tabla 3. Rendimiento forrajero

\begin{tabular}{lccc}
\hline \multirow{2}{*}{ Parámetro } & \multicolumn{3}{c}{ Tratamiento } \\
\cline { 2 - 4 } & $\mathrm{T} 1$ & $\mathrm{~T} 2$ & $\mathrm{~T} 3$ \\
\hline Peso fresco de planta, $\mathrm{kg}$ & $1.01^{\mathrm{a}}$ & $1,08^{\mathrm{a}}$ & $1,05^{\mathrm{a}}$ \\
Peso fresco por hectárea, $\mathrm{t}$ & $68,7^{\mathrm{a}}$ & $73,8^{\mathrm{a}}$ & $71,5^{\mathrm{a}}$ \\
\hline T1: fertilizante químico & & \\
T2: estiércol sólido \\
T3: fertilizante químico + estiércol sólido \\
a Superíndices iguales dentro de filas indican que no hay diferencia \\
estadística (p>0,05)
\end{tabular}

Peso fresco por hectárea. Los pesos obtenidos fueron: 68,$7 ; 73,8$ y 71,5 t/ha para los tratamiento T1, T2 y T3, respectivamente (Tabla 3). En el análisis de variancia no se encontró diferencia significativa $(p>0,05)$ entre los tratamientos; sin embargo numéricamente el tratamiento $\mathrm{T} 2$ supera en $5 \mathrm{t}$ al tratamiento $\mathrm{T} 1$. Los pesos encontrados superan a las $65 \mathrm{t} / \mathrm{ha}$ obtenidos por Fortis et al. (2009) y está dentro del rango esperado, 70 a 75 t/ha, para la variedad utilizada con riego por gravedad, como lo fue en el presente trabajo. El buen resultado obtenido en el T2, posiblemente se deba al alto contenido de $\mathrm{N}$ y materia orgánica que contiene el estiércol porcino seco; asimismo hay que tener en cuenta que el $40 \%$ del $\mathrm{N}$ es orgánico y el $60 \%$ es amoniacal (Moser, 1996). Según FAO (2002), el $\mathrm{N}$ orgánico es de lenta disponibilidad y por lo tanto los cultivos lo pueden ir utilizando a medida que va siendo degradado de formas orgánicas a las formas minerales $\left(\mathrm{NO}_{3}\right)$. Salazar et al. (2007) manifiestan que el incremento en la producción al usar el estiércol no solo es porque retienen la humedad por más tiempo, sino que además es una fuente que libera los nutrientes de manera paulatina a través de todo el ciclo fenológico. Hay una actividad enzimática constante en todo el ciclo, biodegradándolo y liberando iones que están disponibles para las plantas y los microorganismos.

\section{Valor nutricional de la planta}

Los resultados del análisis químico se consignan en la Tabla 4, expresados en base al 100\% de materia seca, valores parecidos a los reportados por Amador y Boschini (2000) ; Gómez (2002); pero significativamente menores en el contenido de proteína; $14,6 \%$, reportado por Castillo et al. (2012). Respecto a la proteína se observa que el tratamiento T3, tiene el valor más alto; $10,5 \%$, superioridad que podría explicarse por la rápida disponibilidad de nutrientes minerales aportados por el fertilizante químico y por el mejoramiento de las propiedades físico, química y biológica del suelo aportado por el estiércol porcino sólido. Asimismo se observa que la fibra cruda es mayor en el tratamiento T2, 28,3\%; lo cual está en concordancia con 
el mayor peso por planta (mayor altura de planta, tamaño de mazorca y diámetro del tallo) que se tuvo en este tratamiento. Estas partes de la planta representan mayor cantidad de fibra total y disminuye el contenido celular soluble (Félix, 2002).

Tabla 4. Análisis proximal del maíz chala

\begin{tabular}{lccc}
\hline Nutrientes & T1 & T2 & T3 \\
\hline Proteína total, \% & 8.9 & 8.7 & 10.5 \\
Grasa, \% & 1.3 & 1.4 & 1.6 \\
Fibra Cruda, \% & 26.2 & 28.3 & 25.4 \\
Extracto Libre de Nitrógeno, \% & 56.9 & 54.5 & 54.0 \\
Fibra Detergente Neutra, \% & 55.6 & 56.3 & 54.8 \\
\hline
\end{tabular}

T1: fertilizante químico

T2: estiércol sólido

T3: fertilizante químico + estiércol sólido

\section{Propiedades fisicoquímicas del suelo}

Las propiedades fisicoquímicas o caracterización del suelo, inicial y post cosecha se muestran en la Tabla 5. El tratamiento que dejo mejores propiedades fisicoquímica del suelo fue el tratamiento T2, materia orgánica 2,56 $\%$, fosforo $59,4 \mathrm{ppm}$ y potasio $230 \mathrm{ppm}$, seguido por el tratamiento T3. La aplicación de materia orgánica mejora la estructura del suelo, aporta y mantiene nutrientes para próximos cultivos (Félix et al., 2008). Menores indicadores dejo el tratamiento T1, lo que corrobora lo mencionado por Díez et al. (2004) donde el fertilizante mineral se lixivia y volatiliza rápidamente a diferencia del orgánico. Asimismo, Fortis et al. (2009) concluyen que la aplicación de abonos orgánicos incrementa la presencia de nitratos lo que permitiría no aplicar nitrógeno al menos al inicio de un nuevo ciclo agrícola.

Tabla 5. Propiedades fisicoquímicas del suelo, inicial y post cosecha

\begin{tabular}{lccccccc}
\hline Muestra & $\begin{array}{c}\mathrm{pH} \\
(1: 1)\end{array}$ & $\begin{array}{c}\mathrm{CE} \\
(1: 1) \\
\mathrm{dS} / \mathrm{m}\end{array}$ & $\begin{array}{c}\mathrm{MO} \\
\%\end{array}$ & $\begin{array}{c}\mathrm{P} \\
\mathrm{ppm}\end{array}$ & $\begin{array}{c}\mathrm{K} \\
\mathrm{ppm}\end{array}$ & $\begin{array}{c}\text { Clase } \\
\text { textural. }\end{array}$ & $\mathrm{CIC}$ \\
\hline Inicial & 6.93 & 3.42 & 1 & 23.5 & 183 & Franco & 21.9 \\
Post cosecha & & & & & & & \\
T1 & 7.04 & 1.47 & 1.01 & 24.7 & 170 & Franco & 19.5 \\
T2 & 7.33 & 1.25 & 2.56 & 59.4 & 230 & Franco & 19.9 \\
T3 & 7.38 & 1.8 & 1.8 & 40.1 & 198 & Franco & 20.8 \\
\hline T1: fertilizante químico & & & & & & \\
T2: estiércol sólido \\
T3: fertilizante químico + estiércol sólido & & & \\
\end{tabular}

\section{Análisis económico}

En el análisis económico por hectárea del maíz chala, medido a través de los costos de producción, ingreso neto e índice de rentabilidad (Tabla 6), se obtuvo un menor costo de producción, S/ 3 858,60; mayor ingreso neto, S/ 4 270,40; y mayor índice de rentabilidad, $111 \%$, en el tratamiento T2. El uso del estiércol seco de porcinos como fertilizante ha influido significativamente en los costos, ya que tiene un valor 10 veces menor que los fertilizantes químicos; asimismo la mayor producción (5 t) de este tratamiento ha influido positivamente en los ingresos. Estos resultados concuerdan con lo aseverado por Cantarero y Martínez (2002) quienes indican que los costos al usar abonos orgánicos son menores, en comparación al uso de fertilizantes sintéticos.

Tabla 6. Análisis económico

\begin{tabular}{ccccc}
\hline Tratamiento & $\begin{array}{c}\text { Ingresos } \\
\text { S/ x ha }\end{array}$ & $\begin{array}{c}\text { Costo } \\
\text { S/ x ha }\end{array}$ & $\begin{array}{c}\text { Ingreso Neto } \\
\text { S/ }\end{array}$ & $\begin{array}{c}\text { Índice } \\
\text { Rentabilidad } \\
\%\end{array}$ \\
\hline T1 & 7557,00 & 4440,80 & 3116,20 & 70 \\
T2 & 8129,00 & 3858,60 & 4270,40 & 111 \\
T3 & 7865,00 & 3901,90 & 3963,10 & 102 \\
\hline
\end{tabular}

T1: fertilizante químico

T2: estiércol sólido

T3: fertilizante químico + estiércol sólido

\section{Conclusiones}

Para las condiciones experimentales y los tratamientos establecidos, el uso del estiércol porcino sólido (T2) usado como abono orgánico produjo estadísticamente $(\mathrm{p}>0,05)$ similar rendimiento de maíz chala que los fertilizantes sintéticos, inclusive con una tendencia numérica positiva (5 t/ha más). Asimismo mejoró las propiedades fisicoquímico del suelo, no afectó el valor nutritivo de la planta, redujo los costos de producción e incrementó la utilidad neta y la rentabilidad. Los resultados permiten evidenciar que la, aplicación de abonos orgánicos, como el estiércol porcino sólido, es una buena alternativa de fertilización, viable, económica y más amigable con el medioambiente.

\section{Literatura citada}

Álvarez, J.; Gómez, D.; León, N. y Gutiérrez, F. 2010. Manejo integrado de fertilizantes y abonos orgánicos en el cultivo de maíz. Agrociencia, 44:575-586.

Amador, A. y Bosochini, C. 2000. Fenología productiva y nutricional de maíz para la producción de forraje. Agronomía Mesoamericana, 11(1):171 - 175.

Asociación Peruana de Porcicultores [APP]. 2018. Negocios: Producción nacional de carne de cerdos aumentó 4\% en 2017. Agencia Agraria de Noticas, enero, 2018.

Cantarero, R. y Martínez, O. 2002. Evaluación de tres tipos de fertilizantes (gallinaza, estiércol vacuno y un fertilizante mineral) en un cultivo de maíz (Zea mays L.). Variedad NB-6. Universidad Nacional Agraria. Managua - Nicaragua. 40-43p

Castillo, C.; Carcelén, F.; Quevedo, W. y Ara, M. 2012. Efecto de la suplementación con bloques minerales sobre la productividad de cuyes alimentados con forraje. Revista de Investigaciones Veterinarias del Perú, 23(4), 414-419.

Díez, J.; Herniáis, P.; Muñoz, A.; De La Torre \& Vallejo, A. 2004. Impact of pig slurry on soil properties, water salinization, nitrate leaching and crop yield in a four-year experiment in Central Spain. Soil use and 
management. Centro de Ciencias Medio Ambientales. Madrid, España.

Eghball, B.; Ginting, D. and Gilley J. 2004. Residual effects of manure and compost applications on corn production and soil properties. Agron. J. 96:442-447.

FAO [Food and Agriculture Organization]. 2016. Producción de carne de cerdo. División de Producción y Sanidad Animal.

FAO [Food and Agriculture Organization]. 2002. Los fertilizantes y su uso.

Félix, R. 2002. Efecto de la densidad de siembra en el rendimiento y valor nutritivo en seis cultivares de maíz chala (DK821, DK834, DK754S, XL650, Chala puente y PM212) para ensilaje en la zona de Chancay (Lima). Tesis Ing. Zoot. Lima, Perú. UNALM, Lima, Perú. 69p.

Félix, J.; Sañudo, R.; Rojo, G.; Martínez, R. and Olalde, V. 2008. Importance of organic manures. Rev. Sociedad, Cultura y Desarrollo. Universidad Autónoma Indígena de México.

Fortis, M.; Leos, J.; Preciado, P.;Orona, I.;García, J. y Orozco, J. 2009. Aplicación de abonos orgánicos en la producción de maíz forrajero con riego por goteo. Terra Latinoamericana, 27:329-336.

Geohring, L.D. and van Es, H.M. 1994. Liquid manure application systems. Design, Management and Environmental Assessment. Proceedings from the liquid manure application system conference. Rochester, New York. Northeast Regional.

Gómez, C. 2002. Avances en alimentación de vacunos lecheros: Valor nutricional y utilización de alimentos. Conferencia - UNALM, 17-20 mayo.

INEI (Instituto Nacional de Estadística e Informática). 2013. IV Censo Nacional Agropecuario 2012. Población ganado porcino, $19 \mathrm{p}$.

López, M. J. D.; Díaz, E. A.; Martínez, R. E. \& Valdés, C. R. D. 2001. Abonos y su efecto en propiedades físicas y químicas del suelo y rendimiento en maíz. Terra Latinoamericana, 19(4), 293-299.

Moser, M. 1996. Estiércol de cerdo: recolección, tratamiento y uso como fertilizante para cultivos. Porcicultura Colombiana. 41:11-19.

Piccinini, S. and Bortone, G. 1991. The fertilizer value of agricultural manure: simple rapid methods of assessment. J. Agric. Eng. Res. 49:197-208.

Pinos, J.; Gracia, J.; Peña, L.; Rendón, J.; Gonzales, C.; y Tristán, F. 2012. Impacto y regulaciones ambientales de estiércol generado por los sistemas ganaderos de algunos países de américa. México. Revista Agrociencia, 46(4):360.

Salazar, E.; Trejo, H.; Vásquez, C y López, J. 2007. Producción de maíz bajo riego por cintilla, con aplicación de estiércol bovino. Rev. Int. Bot. Exp. 76:169-185.

Sotomayor, R.; Chura, J.; Calderón, C.; Sevilla, R y Blas, P.
2017. Fuentes y dosis de nitrógeno en la productividad del maíz amarillo duro bajo dos sistemas de siembra. Anales Científico, 78(2): 232-240. 